\title{
Evaluación formativa en el contexto universitario: oportunidades y propuestas de actuación
}

\author{
Laura Cañadas \\ Universidad Autónoma de Madrid, Facultad de Formación de Profesorado y Educación, \\ Madrid, España \\ https://orcid.org/0000-0003-4179-9018
}

Recibido: 14/07/2020 Revisado:24/10/2020 Aceptado:02/12/2020 Publicado: 10/12/2020

\begin{abstract}
Resumen
La evaluación es uno de los elementos más importantes y a la vez más controvertidos de la formación universitaria. A pesar de la amplia bibliografía que apoya el empleo de los procesos de evaluación formativa en Educación Superior, en la práctica todavía hoy no se da un uso generalizado de esta, predominando los sistemas tradicionales de evaluación que buscan únicamente establecer una calificación al final del periodo formativo. El principal objetivo de este artículo es realizar una revisión sobre las concepciones de la evaluación y mostrar las características que deben tener los procesos de evaluación formativa en Educación Superior, para posteriormente realizar una serie de recomendaciones y propuestas que puedan servir como referencia para el cambio en los procesos de evaluación. Es necesario avanzar hacia la utilización de estos procedimientos de forma sistemática y planificada para conseguir un entorno lo más formativo posible y que únicamente tenga en cuenta la nota como la culminación de este proceso.

Palabras claves: evaluación formativa; educación superior: formación inicial; instrumentos; feedback
\end{abstract}

\section{Formative assessment in university context: opportunities and proposals for action}

\begin{abstract}
Assessment is one of the most important and controversial elements of University training. Despite the extensive bibliography that supports the use of formative assessment processes in Higher Education, in practice, there is still a great predominance in the use of traditional assessment systems that seek to stablish a grade at the end of the training period. The aim of this article is to carry out a review of the concepts of assessment and show the characteristics that formative assessment processes in Higher Education must have, in order to make a series of recommendations and proposals that can serve as reference elements for the change in the assessment processes. It is necessary to advance in the use of these procedures in a systematic and planned way to achieve a more formative environment in a system that keeps a single numeric grade as the culmination of the process.

Keywords: formative assessment; higher education; initial training; instruments: feedback
\end{abstract}




\title{
Avaliação formativa no contexto universitário: oportunidades e propostas de desempenho
}

\begin{abstract}
Resumo
A avaliação é um dos elementos mais importantes e ao mesmo tempo mais controversos do ensino universitário. Apesar da extensa bibliografia que apóia o uso de processos formativos de avaliação no ensino superior, na prática, ainda não há um uso generalizado disso, com sistemas tradicionais de avaliação que buscam certificar a aprendizagem dos alunos através uma nota no final do período de treinamento, geralmente através do uso do exame. Por esse motivo, o principal objetivo deste artigo é realizar uma revisão dos conceitos de avaliação e mostrar as características que os processos de avaliação formativa devem ter, para posteriormente fazer uma série de recomendações e propostas que possam servir como elementos de referência. pela mudança nos processos de avaliação. É preciso caminhar para a utilização desses procedimentos de forma sistemática e planejada para se atingir o ambiente mais educativo possível e que só leve em conta a série como culminância desse processo.
\end{abstract}

Palavras-chave: avaliação formativa; ensino superior: treinamento inicial; instrumentos; feedback.

Citar como:

Cañadas, L. (2020). Evaluación formativa en el contexto universitario: oportunidades y propuestas de actuación. Revista Digital de Investigación en Docencia Universitaria. 14(2), e1214. https://doi.org/10.19083/ridu.2020.1214

\section{Introducción}

La evaluación es uno de los elementos más importantes y a la vez más controvertidos de la formación universitaria. Aunque desde hace años existen numerosas propuestas de evaluación que se orientan a la búsqueda de un aprendizaje más profundo y duradero por parte del alumnado (López-Pastor \& Sicilia-Camacho, 2017), todavía existe una gran reticencia a este cambio, predominando en muchos casos formas tradicionales de evaluación que perpetúan esta como un mero instrumento para calificar al alumnado al final de un periodo formativo (Cañadas, Santos-Pastor, \& Castejón, 2018a; Panadero, Fraile, Fernández-Ruíz, Castilla-Estévez, \& Ruíz, 2019). Entre el profesorado aún permanecen enraizadas muchas concepciones y creencias tradicionales sobre la evaluación, como quién debe evaluar, qué instrumento es el único que realmente vale para asegurar los aprendizajes o la percepción de estos procedimientos como un castigo o elemento sancionador para el alumnado. Todo esto impide un avance generalizado hacia la utilización de formas alter- nativas de evaluación. Esto ha hecho que, en la situación provocada por la COVID-19, hayan surgido numerosas dudas y dificultades para asegurar un proceso evaluativo justo, sostenible y de calidad para el desarrollo de las competencias de las titulaciones. La preocupación generalizada que se ha puesto de manifiesto para tratar de buscar solución a la dificultad de aseverar quién, cómo o con qué recursos se responde a un examen en un contexto de docencia online (Prince, Fulton, \& Garsombke, 2009) ha hecho que se visibilice el gran número de titulaciones y docentes que aún utilizan el examen como principal, y casi como único recurso, para valorar el grado de aprendizaje del alumnado al finalizar el periodo formativo. En Educación Superior la mayoría de las acciones formativas se han encaminado a proporcionar a los docentes herramientas online para poder preparar pruebas de estas características (McDowell, 2020; Nic Dhonncha, \& Murphy, 2020; Rodríguez \& Luzardo, 2020). Esto es indicador del gran peso que sigue teniendo en la evaluación los exámenes finales. Aunque son instrumentos de gran utilidad para asegurar la adquisición de determinadas competencias o aprendizajes, un examen final no 
puede ser el eje sobre el que gire todo un periodo formativo de cuatro meses.

Por ello, todo lo devenido en este periodo debe servirnos como aprendizaje para replantearnos los sistemas de evaluación que venimos empleando. Este cambio no debe ser temporal con objeto de responder a las necesidades de una docencia online, sino que debe plantearse como una necesidad de cambio a largo plazo, convirtiéndose en prácticas perdurables en Educación Superior. Ante esta situación, la formación y evaluación en la Universidad tiene una oportunidad para reinventarse.

El principal objetivo de este artículo es realizar una revisión sobre las concepciones de la evaluación y mostrar las características que deben tener los procesos de evaluación formativa en Educación Superior, para posteriormente realizar una serie de recomendaciones y propuestas que puedan servir como elementos de referencia para el cambio en los procesos de evaluación en Educación Superior.

\section{Concepciones de evaluación en Educación Superior. De la calificación a la evaluación formativa}

Tradicionalmente, la evaluación se orientaba a la rendición de cuentas, a la certificación de unos resultados académicos (Knight, 2006). Su principal objetivo era valorar el grado de consecución de los objetivos previstos al finalizar un periodo de enseñanza (Álvarez, 2011), empleando para ello una calificación como acto que ratifica lo que se aprende (Black, Harrison, Lee, Marshall, \& Wiliam, 2002). Esta concepción se caracterizaba por la búsqueda de objetividad con la finalidad de medir de la forma más fiable posible los productos del alumnado al finalizar el proceso de enseñanza y aprendizaje (Álvarez, 2003). En esta evaluación se equipara evaluar con calificar, limitándose la evaluación a la realización de un examen y siendo el profesorado el único responsable de evaluar y el alumnado el único objeto de la evaluación (Calderón \& Escalera, 2008). Este tipo de evaluación trae como consecuencia que en muchos casos se deje de lado el aprendizaje poniendo el foco de atención en estudiar para el examen.

Sin embargo, a raíz de los cambios estructurales y funcionales que se han producido en la uni- versidad en los últimos 10 años se demanda de otro tipo de evaluación (Benito \& Cruz, 2005). Se demanda de una evaluación para el aprendizaje (Sambell, McDowell, \& Montgomery, 2013). De una evaluación cuya principal función sea la de mejorar los procesos de enseñanza y aprendizaje que tienen lugar en el aula, regulando los aprendizajes para que al finalizar el proceso se consigan los objetivos propuestos en la mayor medida posible (Dolin, Black, Harlen, \& Tiberghien, 2017; Hortigüela, Palacios-Picos, \& López-Pastor, 2019). Este es un tipo de evaluación que debe estar integrada en la práctica docente, en la que tanto el profesorado como el alumnado deben ser objeto de evaluación y donde se debe buscar la mejora del aprendizaje, y no emplear la evaluación como un mero objeto calificador (López-Pastor, 2009).

A pesar de ello, la tradición evaluativa y las prácticas vivenciadas tienen mucho peso, y lleva a que se equipare generalmente evaluar a calificar (López-Pastor, 2017). Muchos de los procesos evaluativos se llevan a cabo de manera que todas las producciones tengan que ir asociadas a una calificación, pero no siempre a una retroalimentación ni a una posterior utilización de esa información para la mejora de los aprendizajes. La evaluación y la calificación tienen finalidades diferentes $\mathrm{y}$, por tanto, no pueden ser utilizadas como si fuesen lo mismo. Hamodi (2016) recoge las diferencias entre estos dos procesos:

- Evaluación: Recogida de información, análisis y emisión de un juicio sobre ella para la toma de decisiones que se desarrolla a lo largo de todo el proceso de enseñanza y aprendizaje. La evaluación debe responder a un aprendizaje continuo (formadora), que permita conocer el progreso del alumnado (reguladora) y mejorar el proceso de enseñanza y aprendizaje (pedagógica) con intercambio de información entre profesorado y alumnado (comunicadora).

- Calificar: Transformar el juicio emitido en una calificación, generalmente al final del proceso. La calificación, principalmente, tiene una función certificadora y de control.

Por ello, aunque la calificación es socialmente necesaria, por las exigencias de certificar que el alumnado ha adquirido un determinado nivel de aprendizajes, no debe ser el eje sobre el que gire 

oportunidades y propuestas de actuación

todo el proceso. Previo al establecimiento de una calificación, debe haberse producido un proceso continuo y formativo de evaluación que haya permitido al alumnado incrementar sus aprendizajes respecto a su nivel inicial y alcanzar los objetivos en el mayor grado posible.

Con objeto de responder ante este necesario cambio surgen diversas propuestas que comparten los principios necesarios para el cambio en los procesos de evaluación. Concretamente, nos centraremos en la evaluación formativa, propuesta que la investigación ha mostrado que repercute positivamente en el desarrollo de competencias, favoreciendo un mayor aprendizaje del alumnado (Barba-Martín, Bores-García, González-Calvo, \& Hortigüela, 2020; Cañadas, Santos-Pastor, \& Castejón, 2020; Gallardo-Fuentes, López-Pastor, \& Carter-Thuillier, 2018; Hortigüela, Fernández-Río, Castejón, \& Pérez-Pueyo, 2017; Romero, Castejón, López-Pastor, \& Fraile-Aranda, 2017).

\section{La evaluación formativa en Educación Superior}

La evaluación formativa puede definirse como la forma en que partiendo de unos criterios previamente establecidos y planificados se recoge información durante el proceso formativo sobre el aprendizaje del alumnado y la enseñanza del profesorado con el objetivo de mejorar el proceso de enseñanza y aprendizaje (Cañadas, 2018; López-Pastor \& Sicilia, 2017). En esta definición se aglutinan algunos de los principales rasgos de los procesos de evaluación formativa, que pueden verse en la Figura 1. A continuación, se describirán las características más relevantes que deben de cumplir los sistemas de evaluación formativa para su efectiva puesta en práctica, y que vienen avaladas por diversas investigaciones (Cañadas, 2018; Wiliam \& Leahy, 2015) (ver Figura 1).

- Sistemática y planificada: Para que la evaluación pueda tener efectos positivos sobre el aprendizaje del alumnado, esta debe estar planificada de antemano, decidiendo qué finalidades se quieren conseguir en el plan o programa, cómo se va a evaluar esto, en qué momentos, de qué forma se va a recoger la información, qué se hará con esa información o quién será el encargado de hacerlo. Además, debe estar integrada en el propio proceso de enseñanza y aprendizaje (López-Pastor, 2017).

- Clarificar los objetivos de aprendizaje y los criterios de evaluación con el alumnado: Uno de los aspectos que delimita la capacidad para conseguir un mayor aprendizaje es

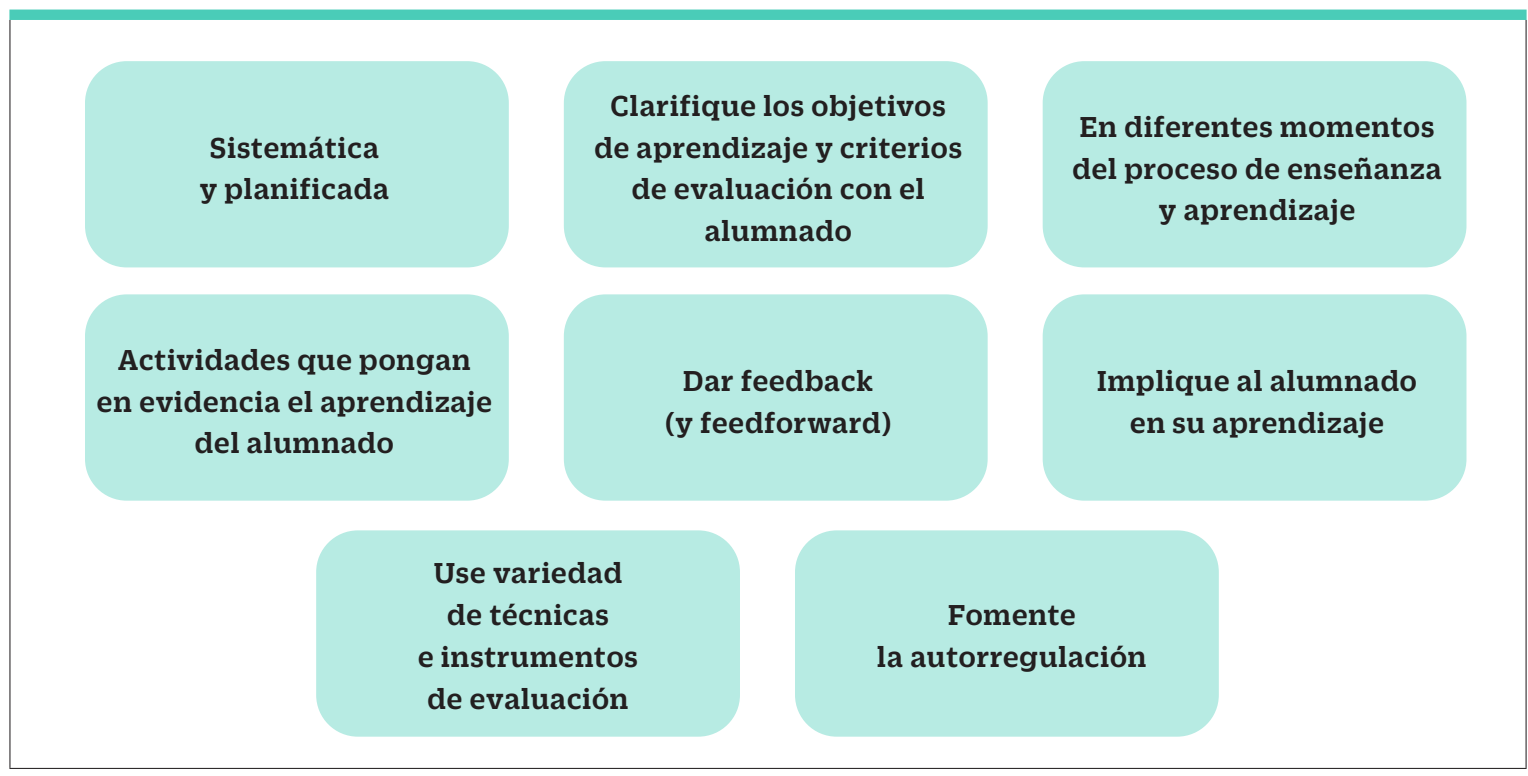

Figura 1. Características de los sistemas de evaluación (Cañadas, 2018). 
el conocimiento previo sobre qué se espera que el alumnado aprenda al finalizar un periodo formativo (Benito \& Cruz, 2005). Para ello es importante compartir con el alumnado tanto los objetivos de aprendizaje como los criterios de evaluación (Villardón, 2006). Esta finalidad de aprendizaje en un sistema de evaluación formativa es la que dirige tanto el feedback como el feedforward que se pueda dar (Frey \& Fisher, 2011). Un paso más en estos procesos sería construir con el alumnado los criterios de evaluación al comienzo del proceso de enseñanza y aprendizaje (Moss \& Brookhart, 2019). De esta forma se hace participe en la creación de esos criterios al alumnado, haciéndole más consciente de qué es lo que debe ser capaz de hacer al finalizar el periodo de aprendizaje, y contribuyendo a que pueda autorregular sus aprendizajes (Moss \& Brookhart, 2019).

- Que se desarrolle a lo largo de diferentes momentos del proceso de enseñanza y aprendizaje: Como exponíamos al comienzo, tradicionalmente la evaluación ha estado asociada a la realización de una prueba al finalizar el periodo formativo con objeto de valorar el grado de adquisición de las competencias y/o aprendizajes. Sin embargo, los procesos de evaluación formativa, para que realmente tenga el impacto deseado sobre el aprendizaje, deben desarrollarse en diferentes momentos del proceso y con diferentes finalidades (Heritage, 2010). Entre estos, encontramos tres momentos: inicial, procesual (o continuo) y final. Generalmente, cada uno de estos momentos tienen una finalidad diferente. A continuación analizaremos brevemente qué finalidades puede adquirir la evaluación en cada uno de estos momentos: (a) Evaluación inicial: Se da al comienzo de un proceso formativo, generalmente con una función diagnóstica, es decir, con la finalidad de conocer cuál es el punto de partida del alumnado con objeto de emplear esta información para adaptar lo programado al nivel del alumnado (Grau \& Gómez, 2010); (b) procesual o continua: tiene lugar a lo largo de todo el proceso de enseñanza y aprendizaje y su función principal es de carácter formativo (Good, 2011). Es decir, busca introducir mejoras en el proceso de enseñanza y aprendizaje (Lukas \& Santiago, 2004); y, (c) la evaluación final, que suele desarrollarse con un carácter sumativo, es decir, busca constatar el grado de adquisición de los aprendizajes desarrollados (Good, 2011). En algunos casos, la evaluación continua puede adquirir un carácter sumativo cuando se emplea para certificar el grado de aprendizaje a lo largo del proceso, generalmente a través de pruebas o calificaciones parciales. De la misma forma, la evaluación final puede adquirir un carácter formativo si se emplea la información obtenida para ajustar los siguientes aprendizajes de un periodo formativo.

- Fomentar actividades que pongan en evidencia el aprendizaje del alumnado: La evaluación, de forma tradicional, se entiende como un proceso de carácter formal con actividades estructuradas y que suele estar establecida en los planes de estudio, buscando evaluar una serie de competencias que deberían haber adquirido en un momento determinado y que, en caso de no haberlo hecho, no permiten pasar al siguiente nivel (Popham, 2013). Sin embargo, dentro de un proceso de evaluación formativa no solo debe tener este carácter, sino que se pueden plantear actividades de evaluación que estén en un punto intermedio donde la evaluación esté planificada por el profesorado para conocer lo que el estudiante sabe y lo que le falta por saber. Por último, también deben existir espacios para una evaluación informal, donde se produzcan encuentros no estructurados entre los agentes implicados en el proceso de enseñanza y aprendizaje y donde se proporcione feedback que permita seguir avanzando (Ruíz-Primo, 2011). Todo esto implica que no todas las actividades de evaluación que se hagan lleven una calificación asociada, de manera que, la evaluación se desvincule de esa perspectiva tradicional y se aplique 
verdaderamente en la búsqueda de una mejora del proceso de enseñanza y aprendizaje (López-Pastor, 2017).

- Dar feedback (y feedforward): Uno de los elementos clave de los procesos de evaluación formativa es la información proporcionada al alumnado sobre cómo lo está haciendo (feedback) y qué debe hacer para mejorar (feedforward). El feedback se define como la información que se transmite sobre lo exitoso que está siendo o ha sido la realización de algo (Cañadas, 2018). Para poder determinar lo exitoso que está siendo es necesario conocer qué se esperaba que fuese capaz de hacer el alumnado en ese momento determinado (Black \& Wiliam, 2009). Una vez que el alumnado es conocedor de en qué punto se encuentra necesita información sobre cómo reducir la distancia entre donde está en ese momento y lo que se espera que sea capaz de hacer. Esa información es la que se conoce como feedforward (Walker, 2013). Además, esta información debe servir para desarrollar procesos de autorregulación en el alumnado, es decir, que poco a poco sea el propio alumno o alumna el que sepa en qué punto está en referencia a esas finalidades de aprendizaje y pueda poner en marcha recursos para acortar esa distancia (Stiggins \& Chappuis, 2005).

Por otra parte, diversos estudios (Gibbs \& Simpson, 2004; Boud \& Molloy, 2013; Nicol \& Macfarlane-Dick, 2004, 2006) han recogido cuáles deben ser las características que tenga un feedback efectivo. Una de las más destacadas es la de Gibbs y Simpson (2004). Para estos autores un feedback efectivo debe: (i) ser suficiente, tanto en lo referente a las veces como a la calidad del mismo; (ii) centrado en la realización del alumnado y su aprendizaje, y no en el propio alumnado; (iii) recibido por el alumnado cuando aún pueden emplear esa información para aprendizajes futuros o recibir apoyos; (iv) apropiado para el propósito de la evaluación y los objetivos o competencias propuestas; (v) apropiado para la comprensión de lo que deberían estar haciendo; (vi) de utilidad, se recibe y se utiliza para mejorar el aprendizaje; y (vii) el alumnado activa el proceso de retroalimentación cambiando su forma de afrontar las tareas futuras.

- Implicar al alumnado en su aprendizaje y en el de sus compañeros/as: Fomentar la participación del alumnado en los procesos de evaluación ayuda a reforzar el carácter formativo de la evaluación y a compartir la responsabilidad de dicho proceso con ellos (Adachi, Tai, \& Dawson, 2018; Ibarra, Rodríguez, \& Gómez, 2012; Rodríguez, Ibarra, \& García, 2013). La participación del alumnado se puede producir a partir de procesos de autoevaluación y coevaluación o evaluación entre iguales. La primera hace referencia al proceso por el que un estudiante juzga su propio trabajo (Andrade, 2019); mientras que la coevaluación o evaluación entre pares alude al proceso por el que una persona juzga el trabajo de otra facilitándole feedback sobre su desempeño (López-Pastor, 2017). En el caso de la evaluación entre iguales, el alumnado puede asumir el rol de evaluador o evaluado (Li, 2017). Cuando actúa como evaluador su función será la de identificar en base a unos criterios (internos o externos) la calidad del trabajo de otro compañero o compañera y dar feedback sobre su desempeño (Li, Liu, \& Steckelberg, 2010). La evaluación entre iguales también puede ser individual o grupal, y esta última a su vez puede ser intrergrupal haciendo referencia a la evaluación de un grupo a otro, y la intragrupal, que es el proceso en el que se evalúan los participantes entre ellos dentro de su grupo (Hamodi, 2016). La implicación del alumnado en el proceso de evaluación tendrá una doble función. Por un lado, como medio para desarrollar los procesos de autorregulación de los aprendizajes, es decir, el control que la persona tiene sobre sus acciones, pensamientos o emociones para alcanzar sus aprendizajes (Panadero, Jonsson, \& Botella, 2017; Zimmerman, 2000); $\mathrm{y}$, por otro lado, como práctica democráti- 
ca que implica al alumnado en su propio aprendizaje a través de procesos de respeto y responsabilidad compartida (López-Pastor, 2009). En los procesos de evaluación que participa el alumnado, encontramos otra modalidad de evaluación. Esta es la evaluación compartida. La evaluación compartida se define como los procesos de diálogo entre el profesorado y el alumnado sobre la evaluación (López-Pastor, et al., 2007). Por último, los procesos de participación del alumnado en la evaluación pueden verse culminados por su participación en la calificación. En este caso, de la misma forma que en los procesos de evaluación, la participación del alumnado en la calificación puede ser a través de la auto-calificación (ponerse una nota a uno mismo), la calificación entre iguales (asignar una calificación a un compañero o compañera) y la calificación dialogada (proceso en el que se establece de forma dialogada entre el profesorado y el alumnado la nota) (López-Pastor, 2012).

- Que emplee variedad de técnicas e instrumentos de evaluación: Cuando hablamos de evaluar el aprendizaje del alumnado, hablamos de evaluar este en todos sus ámbitos de desarrollo (Cañadas, Santos-Pastor, \& Castejón, 2018b). Generalmente, en el contexto de Educación Superior actual hablamos de evaluar las competencias desarrolladas en una asignatura o periodo formativo, lo que implicar tener que evaluar finalidades de aprendizaje que se dirigen al ámbito cognitivo, procedimental, social y emocional de las personas (Frost, de Pont, \& Brailsford, 2012; García \& Morillas, 2011). Para ello, en los contextos formativos se han ido desarrollando cada vez una mayor variedad de técnicas e instrumentos de evaluación que permiten recoger el aprendizaje del alumnado (Tillema \& Smith, 2009). Una técnica de evaluación se entiende como las estrategias que se utilizan para la recogida de la información (Rodríguez \& Ibarra, 2011). Por su parte los instrumentos de evaluación se definen como procedimientos estandarizados que aportan información objetiva sobre el desempeño de una persona (Muñiz \& Fonseca-Pedrero, 2008). Tanto las técnicas como los instrumentos de evaluación deben seleccionarse en función de diferentes aspectos: finalidades de aprendizaje, alumnado participante de la asignatura, coherencia con la metodología empleada, etc. (Castejón, Capllonch, González-Fernández, \& López-Pastor, 2009). Son numerosas las técnicas e instrumentos de evaluación existentes que han mostrado ser de gran utilidad para evaluar los aprendizajes adquiridos por el alumnado. En la Tabla 1 puede encontrarse una relación de estos.

Un elemento transversal a todas estas características, y que ha salido en la mayoría de ellas, es la regulación y auto-regulación de los aprendizajes. Los procesos de evaluación formativa son fundamentales para desarrollar en el alumnado la capacidad de aprender a aprender, y para ello es necesario desarrollar la capacidad auto-regular los aprendizajes. La auto-regulación es un proceso a través del cual el alumnado representa mentalmente el objetivo a conseguir y asegura que se alcanzan los objetivos establecidos (Efklides, 2011). Este proceso consta de varias fases de carácter cíclico: planifica cómo llevarla a cabo, realiza un seguimiento sobre si su realización es adecuada, evalúa su desempeño y analiza la causa de su resultado (Panadero, Alonso-Tapia \& Huertas, 2012). Tanto la sistematicidad del proceso, como compartir los objetivos y criterios de evaluación (o construirlos con el alumnado), que se desarrollen actividades que permitan ir valorando parcialmente el desempeño del alumnado, introducir cambios en el proceso de enseñanza y aprendizaje si fuese necesario, y, muy importante, implicar al alumnado en la evaluación y generar oportunidades para recibir (de forma externa o interna) feedback sobre cómo está siendo el desempeño e información sobre qué debe hacerse para mejorar contribuirán a esa auto-regulación de los aprendizajes".

\section{Criterios de calidad de los sistemas de evaluación formativa}

Encontramos una serie de criterios que debe cumplir cualquier sistema de evaluación formativa que quiera ponerse en práctica y que aseguren su 
Tabla 1.

Métodos, técnicas e instrumentos de evaluación según Hamodi (2014, p.24)

\begin{tabular}{|c|c|c|c|c|c|}
\hline \multirow{9}{*}{ 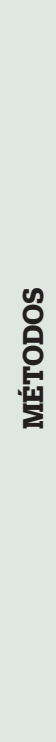 } & \multirow{5}{*}{ Escritos } & Carpeta o dossier & Estudio de casos & Portafolio/ electrónico & Control (examen) \\
\hline & & Ensayo & Poster & $\begin{array}{l}\text { Cuaderno/ de notas/de } \\
\text { campo }\end{array}$ & Foro virtual \\
\hline & & Proyecto & Cuestionario & Memoria & Prueba objetiva \\
\hline & & $\begin{array}{l}\text { Diario/ reflexivo/ de } \\
\text { clase }\end{array}$ & monografía & Recensión & Informe \\
\hline & & \multicolumn{2}{|c|}{ Test de diagnóstico } & \multicolumn{2}{|c|}{ Trabajo escrito } \\
\hline & \multirow{3}{*}{ Orales } & Comunicación & Exposición & Ponencia & Cuestionario oral \\
\hline & & Discusión grupal & Pregunta de clase & \multicolumn{2}{|c|}{ Debate/ diálogo grupal } \\
\hline & & \multicolumn{2}{|c|}{ Presentación oral } & \multicolumn{2}{|c|}{ Mesa redonda } \\
\hline & Prácticos & Práctica supervisada & \multicolumn{2}{|c|}{ Demostración, actuación o representación } & Role-Playing \\
\hline \multirow{5}{*}{ 怘 } & \multirow{2}{*}{$\begin{array}{c}\text { Sin la } \\
\text { participación } \\
\text { del alumnado }\end{array}$} & \multicolumn{4}{|c|}{ Análisis documental y de producciones (revisión de trabajos personales y grupales) } \\
\hline & & \multicolumn{4}{|c|}{$\begin{array}{l}\text { Observación, observación directa del alumno, observación del grupo, observación sistemática, } \\
\text { análisis de grabación de audio o video }\end{array}$} \\
\hline & \multirow{3}{*}{$\begin{array}{c}\text { Con la } \\
\text { participación } \\
\text { del alumnado }\end{array}$} & \multicolumn{4}{|c|}{ Autoevaluación (mediante la autorreflexión y/o el análisis documental) } \\
\hline & & \multicolumn{4}{|c|}{ Evaluación entre iguales (mediante el análisis documental y/o la observación) } \\
\hline & & \multicolumn{4}{|c|}{$\begin{array}{l}\text { Evaluación compartida (mediante una entrevista individual } \\
\text { o grupal entre el profesorado y el alumnado) }\end{array}$} \\
\hline \multirow{3}{*}{ 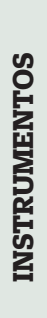 } & $\begin{array}{l}\text { Diario del } \\
\text { profesor/a }\end{array}$ & Escala de estimación & $\begin{array}{c}\text { Fichas de } \\
\text { autoevaluación }\end{array}$ & $\begin{array}{l}\text { Escala de } \\
\text { comprobación }\end{array}$ & $\begin{array}{l}\text { Fichas de } \\
\text { observación }\end{array}$ \\
\hline & Lista de control & $\begin{array}{l}\text { Fichas de evaluación } \\
\text { entre iguales }\end{array}$ & $\begin{array}{l}\text { Escala de diferencial } \\
\text { semántico }\end{array}$ & $\begin{array}{l}\text { Escala verbal o } \\
\text { numérica }\end{array}$ & Matrices de decisión \\
\hline & $\begin{array}{l}\text { Informe de } \\
\text { expertos }\end{array}$ & $\begin{array}{l}\text { Escala descriptiva o } \\
\text { rúbrica }\end{array}$ & Fichas de seguimiento & $\begin{array}{l}\text { Informe de } \\
\text { autoevaluación }\end{array}$ & \\
\hline
\end{tabular}

calidad teniendo en cuenta el contexto en el que se quiera aplicar. Estos criterios son (López-Pastor, 2006, 2008, 2009, 2017):

- Adecuación: El sistema de evaluación propuesto debe estar acorde a las competencias o finalidades de la asignatura y en concordancia con la metodología que se emplee.

- Formativa: Que la evaluación sirva para mejorar y aprender más.

- Ética: Hace referencia a, por un lado, asegu- rar la confidencialidad de la información recabada a través de las pruebas de evaluación; y, por otro lado, y muy importante, a no usar la calificación como elemento de poder y control que permita sancionar al alumnado.

- $\quad$ Relevante: Elegir, entre todos los posibles elementos a evaluar, los más relevantes del proceso que nos den información concreta y suficiente para conocer si se han adquirido las competencias propuestas. 
- Integrada: Hace referencia a la necesidad de que la evaluación sea parte intrínseca del proceso de enseñanza y aprendizaje, y no un elemento externo y descontextualizado de las actividades. Además, debe implicar al alumnado, y en los procesos de evaluación debe integrar las diferentes competencias y contenidos trabajados.

- Veracidad: Disponer de criterios objetivos para la valoración del aprendizaje del alumnado, y de suficientes evidencias que permitan recoger ampliamente el aprendizaje del alumnado, sin que este quede limitado a la información recogida en una única prueba.

- Viable: Que pueda llevarse a cabo, que esté equilibrado y no suponga una carga de trabajo inasumible ni para el profesorado ni el alumnado. Los aspectos propuestos para la evaluación deben tener en cuenta el tiempo disponible tanto por profesorado como alumnado, el número de estudiantes, etc.

\section{Propuestas para la implementación de procesos de evaluación formativa en la Educación Superior}

Los cambios en los procesos evaluativos en Educación Superior deben introducirse de forma gradual. Introducir todas las posibles acciones de golpe únicamente puede derivar en dificultades del profesorado para llevarlas a la práctica, sobrecarga de trabajo por no poder adecuar la carga de trabajo que suponen estos sistemas de evaluación, y dificultades del alumnado para adaptarse a los mismos por el desconocimiento. La falta de formación en estos tipos de procesos en muchos casos lleva a confundir lo que significa implementar procesos de evaluación formativa igualando estos al simple hecho de introducir pruebas parciales o entregas de trabajos escritos para calificar al alumnado (Cañadas, 2018). Por ello, es importante conocer propuestas concretas de cambio que permitan poco a poco ir transformando los sistemas de evaluación en la universidad:

- Analizar las competencias de las titulaciones valorando su tipología: El primer paso para poder cambiar la forma en que evaluamos es realizar un análisis de las competencias asociadas a nuestras titulaciones y asignaturas. De esta forma podremos determinar claramente qué debe haber desarrollado el alumnado al concluir un proceso formativo, y, por tanto, qué conjunto de acciones evaluativas podrían ser empleadas para valorar el grado de adquisición de dichos aprendizajes.

- Programar actividades de enseñanza-aprendizaje-evaluación: Otro de los elementos a cambiar es conseguir plantear actividades de evaluación integradas en el proceso de enseñanza y aprendizaje que no necesariamente tengan que estar asociadas con procesos de calificación, de manera que se pueda recoger información que permita valorar en qué punto está el alumnado, qué aspectos habría que cambiar o mejorar, y que reciban feedback (propio o externo) sobre su desempeño (López-Pastor, 2017). Para ello, en un contexto de formación online deben emplearse actividades de evaluación síncronas (cuestionarios a través de Apps, juegos de pregunta-respuesta, debates, etc.) que permitan valorar y comentar con el alumnado en qué punto están con respecto a los objetivos de aprendizaje. En un contexto de docencia presencial deben desarrollarse tareas presencialmente con el alumnado y contextualizadas que permitan analizar qué han aprendido con respecto a lo esperado en un momento determinado y darles feedback para mejorar (Cano, 2008). El tipo de tareas no tiene porqué variar de un contexto a otro.

- Tareas equilibradas en tiempo de trabajo para el alumnado y el profesorado: Una de las mayores dificultades al comenzar a emplear procesos de evaluación formativa es la dificultad para equilibrar el tiempo de trabajo (Vallés, Ureña, \& Ruíz, 2011). En la mayoría de las ocasiones se tiende a pensar que implementar procesos de evaluación formativa es igual a mandar muchas tareas al alumnado, lo que por un lado dificultad un correcto desempeño del alumnado por sobrecarga de trabajo y por otro impide al profesorado poder dar una adecuada re- 
troalimentación que les permita avanzar en su aprendizaje. Por ello, debe haber una correcta planificación de aquellas tareas con un carácter formal y que van a servir para registrar el desempeño del alumnado acompañándolo de procesos de retroalimentación, y aquellas con un carácter más informal que van a servir para valorar de forma general en el grupo-clase cómo se están desarrollando los aprendizajes y retroalimentar al alumnado durante el proceso tratando de mejorar de forma global sus aprendizajes, y no únicamente su desempeño en una tarea concreta.

- Fomentar la utilización de actividades de auto-evaluación y evaluación entre iguales: Es importante, en primer lugar, conocer la experiencia del alumnado en estos procesos para poder tomar decisiones sobre el grado de autonomía que les damos en el proceso. Es decir, si les damos más o menos ayudas (rúbricas, indicadores concretos, etc.) para llevar a cabo su evaluación. Para ello, cuando el alumnado no haya trabajado previamente partiremos de procesos donde se expliquen los criterios de evaluación al alumnado, y se les acompañe en el trabajo, ayudándoles a identificar los elementos a valorar. Podrán emplearse instrumentos como rúbricas de evaluación, ya que desgranan de forma muy concreta los criterios de evaluación en aspectos observables e identificables. A medida que el sujeto vaya ganando experiencia en estas prácticas habrá que irle quitando ayudas, y facilitando procesos donde ellos sean quienes construyan los criterios de evaluación de tareas o actividades concretas para valorar el desempeño de sus compañeros y compañeras. Además, deberá evolucionarse de procesos donde el alumnado informa sobre qué faltaba a una tarea concreta para ser correcta a procesos donde indiquen qué cosas se podría haber hecho para mejorarlas. Por ello, estos procesos no pueden ser lanzados al alumnado si no hay un proceso previo de enseñanza sobre cómo realizarlo (Panadero \& Alonso-Tapia, 2011). Tan- to en contextos de docencia online como docencia presencial, deben emplearse las herramientas a nuestra disposición para fomentar la participación del alumnado en los procesos de evaluación.

- Apertura hacia nuevos instrumentos de evaluación y su utilización en diferentes momentos del proceso: Uno de los principales elementos de cambio debe ser la programación de la utilización de varios instrumentos de evaluación a lo largo del proceso que permitan valorar la adquisición de competencias en toda su amplitud (Cano, 2008). Son numerosos los instrumentos que permiten la recogida de evidencias a lo largo del curso. Actualmente, destaca la incorporación de aplicaciones móviles en este proceso, como facilitadores de la recogida de información e implicación del alumnado en el proceso.

- Crear de forma compartida con el alumnado los criterios e instrumentos de evaluación: En ese proceso de dar autonomía al alumnado, de fomentar su participación y desarrollar procesos de auto-regulación, se debe fomentar que el alumnado construya junto al profesorado los criterios de evaluación de una tarea concreta o de la asignatura, y junto con ello sea participe de la elaboración de los instrumentos de evaluación que se empleen para ello (Fraile, 2018). Esto puede realizarse al comienzo de curso a través de una asamblea con el alumnado o, si el alumnado no tiene experiencia en este tipo de procesos, puede realizarse de forma gradual en las diferentes tareas de la asignatura.

- Emplear tiempo al comienzo de la clase para recordar lo trabajado previamente y su relación con los objetivos de aprendizaje: Dedicar un breve espacio de tiempo al comienzo de la clase para recordar lo trabajado en sesiones anteriores (Monereo, 1990). Para ello, se pueden emplear procedimientos que permitan una participación conjunta de todo el grupo-clase y que den 
una idea de lo asimilado hasta ese momento. Además, esto permitirá hacer conexiones al alumnado entre lo aprendido y lo que va a aprender, construyendo un aprendizaje más sólido y duradero en el tiempo.

\section{Conclusión}

La investigación ha mostrado la gran utilidad de los procesos de evaluación formativa en la Educación Superior. La aplicación de alguna o todos los elementos indicados en este trabajo han sido destacados como relevantes para el desarrollo de competencias y el aprendizaje del alumnado en este contexto. Por ello, es necesario avanzar hacia la utilización de estos procedimientos de una forma sistemática y planificada para conseguir un entorno lo más formativo posible y que descentralice los procesos de calificación centrándose en conseguir el mayor aprendizaje posible, y únicamente tenga en cuenta la nota como la culminación de este proceso.

\section{Referencias}

Adachi, C., Tai, J., \& Dawson, P. (2018). Academics' perceptions of the benefits and challenges of self and peer assessment in higher education. Assessment \& Evaluation in Higher Education, 43(2), 294-306. https:// doi.org/10.1080/02602938.2017.1339775

Álvarez, J.M. (2003). La evaluación a examen. Ensayos críticos. Buenos Aires: Miño y Dávila.

Álvarez, J.M. (2011). Evaluar para conocer. Examinar para excluir. Madrid: Morata.

Andrade, H. (2019). A Critical Review of Research on Student Self-Assessment. Frontiers in Education, 4(87). https://doi.org/10.3389/feduc.2019.00087

Barba-Martín, R.A., Bores-García, D., González-Calvo, G., y Hortigüela, D. (2020). Evaluación formativa con los estudiantes en prácticas para reducir la brecha teoría-práctica en la formación inicial del profesorado. Educación Física y Deporte, 39(1). http://doi. org/10.17533/udea.efyd.v39n1a02
Benito, A., y Cruz, A. (2005). Nuevas claves para la docencia universitaria en el Espacio Europeo de Educación Superior Madrid: Narcea.

Black, P., Harrison, C., Lee, C., Marshall, B., \& Wiliam, D. (2002). Working inside the black box: assessment for learning in the classroom. London: King's College.

Black, P., \& Wiliam, D. (2009). Developing the theory of formative assessment. Educational Assessment, Evaluation and Accountability, 21(5). https://doi. org/10.1007/s11092-008-9068-5

Boud, D., \& Molloy, E. (2013). Rethinking models of feedback for learning: the challenge of design. Assessment \& Evaluation in Higher Education, 38(6), 698-712. https://doi.org/10.1080/02602938.2012.691462

Calderón, C., y Escalera, G. (2008). La evaluación de la docencia ante el reto del Espacio Europeo de Educación Superior. Educación XX1, 11, 237-256. https:// doi.org/10.5944/educxx1.11.0.316

Cano, E. (2008). La evaluación por competencias en Educación Superior. Profesorado. Revista de Currículum y Formación de Profesorado, 12(3), 1-16.

Cañadas, L. (2018). La evaluación formativa en la adquisición de competencias docentes en la formación inicial del profesorado de Educación Física. Tesis Doctoral Inédita. Madrid: Universidad Autónoma de Madrid.

Cañadas, L., Santos-Pastor, M.L., y Castejón, F.J. (2018a). Evaluación en la formación inicial: ¿avance o retroceso? Bordón, Revista de Pedagogía, 70(4), 9-22. https://doi.org/10.13042/Bordon.2018.64434

Cañadas, L., Santos-Pastor, M.L., y Castejón, F.J. (2018b). Desarrollo de Competencias Docentes en la Formación Inicial del Profesorado de Educación Física. Relación con los Instrumentos de Evaluación. Es tudios Pedagógicos, 44(2), 111-126. 10.4067/S071807052018000200111

Cañadas, L., Santos-Pastor, M.L., y Castejón, F.J. (2020). Competencias del profesorado de Educación Física y valoración en la práctica profesional. Apunts. Educación Física y Deporte, 139, 33-41. https://doi. org/10.5672/apunts.2014-0983.(2020/1).139.05

Castejón, J., Capllonch, M., González-Fernández, N., y López-Pastor, V.M. (2009). Técnicas e instrumentos de evaluación formativa y compartida para la docencia universitaria. En V.M. López-Pastor. (coord.). Evaluación formativa y compartida en educación superior (pp. 65-91). Madrid: Narcea.

Dolin, J., Black, P., Harlen, W., \& Tiberghien, A. (2018). Explo- 
ring Relations Between Formative and Summative Assessment. In J. Dolin \& R. Evans (Ed). Transforming Assessment (pp. 53-80). Cham, Switzerland: Springer International Publishing.

Efklides, A. (2011). Interactions of metacognition with motivation and affect in self-regulated learning: The MASRL model. Educational Psychologist, 46(1), 6-25. https://doi.org/10.1080/00461520.2011.538645

Fraile, J. (2018). La autoevaluación en el Grado de Ciencias de la Actividad Física y del Deporte: concepciones y práctica de los docentes. Tesis Doctoral Inédita. Madrid: Universidad Politécnica de Madrid.

Frey, N., \& Fisher, D. (2011). The formative assessment action plan. Practical Steps to More Successful Teaching and Learning. Estados Unidos: ASCD.

Frost, J., De Pont, G., \& Brailsford, I. (2012). Expanding assessment methods and moments in history. Assessment \& Evaluation in Higher Education, 37(3), 293304. https://doi.org/10.1080/02602938.2010.531247

Gallardo-Fuentes, F., López-Pastor, V.M., y Carter-Thuillier, B. (2018). Efectos de la Aplicación de un Sistema de Evaluación Formativa en la Autopercepción de Competencias Adquiridas en Formación Inicial del Profesorado. Estudios pedagógicos, 44(2), 55-77. http:// dx.doi.org/10.4067/S0718-07052018000200055

García, M.P., y Morillas, L.R. (2011). La planificación de evaluación de competencias en Educación Superior. REIFOP, 14(1), 113-124.

Gibbs, G., \& Simpson, C. (2004). Conditions under which assessment supports students' learning. Learning and Teaching in Higher Education, 1, 3-31. Recuperado de: http://eprints.glos.ac.uk/3609/ el 20 de abril de 2020.

Good, R. (2011) Formative Use of Assessment Information: It's a Process, So Let's Say What We Mean. Practical Assessment, Research, and Evaluation, 16(3). https://doi.org/10.7275/3yvy-at83

Grau, S., y Gómez, M. C. (2010). La evaluación, un proceso de cambio para el aprendizaje. En M.C. Gómez., y S. Grau. (Coords). Evaluación de los aprendizajes en el Espacio Europeo de Educación Superior (pp. 17-33). Alicante: Universidad de Alicante.

Hamodi, C. (2014). La evaluación formativa y compartida en Educación Superior: un estudio de caso. Valladolid: Universidad de Valladolid. Tesis inédita.

Hamodi, C. (2016). Terminología básica sobre evaluación del aprendizaje. En C. Hamodi. (Coord). Formar mediante la evaluación en la Universidad. Propuestas prácticas útiles para docentes (pp. 23-33). Vallado- lid: Ediciones de la Universidad de Valladolid.

Heritage, M. (2010). Formative Assessment: Making it happen in the classroom. Estados Unidos: SAGE.

Hortigüela., D., Fernández-Río, J., Castejón, J. \& Pérez-Pueyo, A. (2017). Formative assessment, work regulation, organization, engagement, tracking and attendance in Spanish Universities. Revista Elec trónica Interuniversitaria de Formación del Profesorado, 20(3), 49-63. http://dx.doi.org/10.6018/reifop.20.3.268681

Hortigüela, D., Palacios-Picos, A., \& López-Pastor, V.M (2019). The impact of formative and shared or co-as sessment on the acquisition of transversal competences in higher education. Assessment \& Evalua tion in Higher Education, 44(6), 933-945. https://doi. org/10.1080/02602938.2018.1530341

Ibarra, M.S., Rodríguez, G. y Gómez, M. A. (2012). La evaluación entre iguales: beneficios y estrategias para su práctica en la universidad. Revista de Educación, 359, 206-231. https://doi.org/10.4438/1988-592XRE-2011-359-092

Knight, P. (2006). The local practices of assessment. Assessment and Evaluation in $\mathrm{Hi}$ gher Education, 31(4), 435-452. https://doi. org/10.1080/02602930600679126

Li, L. (2017). The role of anonymity in peer assessment. Assessment \& Evaluation in Higher Education, 42(4), 645-656. https://doi.org/10.1080/02602938.2016.117 4766

Li, L., Liu, X., \& Steckelberg, A.L. (2010). Assessor or assessee: How student learning improves by giving and receiving peer feedback. British Journal of Educational Technology, 41(3), 525-536. https://doi.org/10.1111/j.1467-8535.2009.00968.x

López-Pastor, V.M. (2006). El papel de la evaluación formativa en el proceso de convergencia hacia el E.E.E.S. Análisis del estado de la cuestión y presentación de un sistema de intervención. Revista Interuniversitaria de Formación del Profesorado, 20(3), 93-119

López-Pastor, V.M. (2008). Desarrollando sistemas de evaluación formativa y compartida en la docencia universitaria. Análisis de resultados de su puesta en práctica en la formación inicial del profesorado. European Journal of Teacher Education, 31(3), 293-311. https://doi.org/10.1080/02619760802208452

López-Pastor, V. M. (Coord.) (2009). La evaluación Formativa y Compartida en Educación Superior: propues tas, técnicas, instrumentos y experiencias. Madrid: Narcea. 
López-Pastor, V.M. (2012). Evaluación Formativa y Compartida en la Universidad: Clarificación de Conceptos y Propuestas de Intervención desde la Red Interuniversitaria de Evaluación Formativa. Psychology, Society \& Education, 4(1). http://dx.doi.org/10.25115/ psye.v4i1.485

López-Pastor, V. M. (2017). Evaluación formativa y compartida: evaluar para aprender y la implicación del alumnado en los procesos de evaluación y aprendizaje. En V.M. López-Pastor \& Á. Pérez-Pueyo (Coords). Evaluación formativa y compartida en educación: experiencias de éxito en todas las etapas educativas (pp. 34-68). León: Universidad de León.

López-Pastor, V.M., Barba-Martín, J.J., Monjas-Aguado, R., Manrique-Arribas., Heras-Bernardino, C., González-Pascual, M., y Gómez-García, J.M. (2007). Trece años de Evaluación Compartida en Educación Física. Revista Internacional en Medicina y Ciencias de la Actividad Física y el Deporte, 7(26), 69-86.

López-Pastor, V.M., y Sicilia Camacho, A. (2017). Formative and shared assessment in higher education. Lessons learned and challenges for the future. Assessment \& Evaluation in Higher Education, 42(1), 77-97. https://doi.org/10.1080/02602938.2015.1083535

Lukas, J.F., \& Santiago, K. (2004). Evaluación Educativa. Madrid: Alianza Editorial.

McDowell, S. (2020). Asynchronous Online Assessment of Physical Chemistry Concepts in the Time of COVID-19. Journal of Chemical Education, 97, 3256-3259. https://dx.doi.org/10.1021/acs.jchemed.0c00611

Monereo, C. (1990). Las estrategias de aprendizaje en la Educación Formal: enseñar a pensar y sobre el pensar. Infancia y Aprendizaje, 50, 3-25.

Moss, C.M., \& Brookhart, S.M. (2019). Advancing Formative Assessment in Every Classroom: A Guide for Instructional Leaders. United States: ASCD

Nic Dhonncha, E., \& Murphy, M. (2020). Learning new ways of teaching and assessment: the impact of COVID-19 on undergraduate dermatology education. Clinical and Experimental Dermatology. https:// doi.org/10.1111/ced.14364

Nicol D.J. \& Macfarlane-Dick, D. (2004). Rethinking Formative Assessment in HE: a theoretical model and seven principles of good feedback practice. http:// www.heacademy.ac.uk/assessment/ASS051D_SENLEF_model.doc

Nicol, D.J., \& Macfarlane-Dick, D. (2006). Formative assess- ment and sel-regulated learning: a model and seven principles of good feedback practice. Studies in Higher Education, 31(2), 199-218. https://doi. org/10.1080/03075070600572090

Panadero, E., y Alonso-Tapia, J. (2011). El papel de las rúbricas en la autoevaluación y autorregulación del aprendizaje. En K. Bujan., I. Rekalde., y P. Aramendi. (Eds). La evaluación de competencias en la Educación Superior, Las rúbricas como instrumento de evaluación. Madrid: Eduforma.

Panadero, E., Alonso-Tapia, J., \& Huertas, J.A. (2011). Rubrics and self-assessment scripts effects on self-regulation, learning and self-efficacy in secondary education. Learning and Individual Differences, 22(6), 806-813. https://doi.org/10.1016/j.lindif.2012.04.007

Panadero, E., Fraile, J., Fernández-Ruíz, J., Castilla-Estévez, D., \& Ruíz, M.A. (2019). Spanish university assessment practices: examination tradition with diversity by faculty. Assessment \& Evaluation in Higher Education, 44(3), 279-295. https://doi.org/10.1080/0 2602938.2018.1512553

Panadero, E., Jonsson, A., \& Botella, J. (2017). Effects of self-regulated learning and self-efficacy: Four meata-analyses. Educational Research Review, 22, 74-98. https://doi.org/10.1016/j.edurev.2017.08.004

Popham, W.J. (2013). Evaluación trans-formativa. El poder transformador de la evaluación. Madrid: Narcea.

Prince, D. J., Fulton, R. A. \& Garsombke, T. W. (2009). Comparisons of proctored versus non-proctored testing strategies in graduate distance education curriculum. Journal of College Teaching \& Learning, 6(7), 51-63. https://doi.org/10.19030/tlc.v6i7.1125

Rodríguez, G., e Ibarra, M. S. (2011). E-Evaluación orientada al e-aprendizaje estratégico en Educación Superior. Madrid: Narcea.

Rodríguez, G., Ibarra, M. S. y García, E. (2013). Autoevaluación, evaluación entre iguales y coevaluación: Conceptualización y práctica en las universidades españolas. Revista de Investigación en Educación, 11(2), 198-210.

Rodríguez, P., y Luzardo, M. (2020). Cómo Asegurar Evaluaciones Válidas y Detectar Falseamiento en Pruebas a Distancia Síncronas. RIDU. Revista Digital de Investigación en Docencia Universitaria, 14(2), e1240. https://doi.org/10.19083/ridu.2020.1240

Romero, M.R., Castejón, F.J., López-Pastor, V.M., y Fraile-Aranda, A. (2017). Evaluación formativa, competencias comunicativas y TIC en la Formación del Profesorado. Comunicar. 52(3), 73-82. https://doi. 
org/10.3916/C52-2017-07

Ruíz-Primo, M.A. (2011). Informal formative assessment: The role of instructional dialogues in assessing students' learning. Studies in Educational Evaluation, 37, 15-24. https://doi.org/10.1016/j.stueduc.2011.04.003

Sambell, K., McDowell, L., \& Montgomery, C. (2013). Assess ment for Learning in Higher Education. Abingdon, U. K.: Routledge.

Stiggins, R., \& Chappuis, J. (2005). Using Student-Involved Classroom Assessment to Close Achievement Gaps. Theory Into Practice, 44(1), 11-18. https://doi. org/10.1207/s15430421tip4401_3

Tillema, H.H., \& Smith, K. (2009). Assessment orientation in formative assessment of learning to teach. Teachers and Teaching: theory practice, 15(3), 391-405. https://doi.org/10.1080/13540600903056726

Vallés, C., Ureña, N., y Ruíz, E. (2011). La Evaluación Formativa en Docencia Universitaria. Resultados globales de 41 estudios de caso. REDU. Revista de Docencia Universitaria, 9(1), 135-158.

Villardón, L. (2006). Evaluación del aprendizaje para promover el desarrollo de competencias. Educatio siglo XXI, 24, 57-76.

Walker, M. (2013). Feedback and Feedforward. Student res ponses and their implications. En: S. Merry., M. Price., D. Carless., \& M. Taras (Eds). Reconceptualising Feedback in Higher Education. Developing Dialogue with Students (pp. 103-113). Estados Unidos: Routledge.

Wiliam, D., \& Leahy, S. (2015). Embedding formative assess ment: Practical techniques for $\mathrm{K}-12$ classrooms. West Palm Beach, FL: Learning Sciences International.

Zimmerman, B. J. (2000). Attaining self-regulation: A social cognitive perspective. En M. Boekaerts, P. R. Pintrich \& M. Zeidner (Eds.), Handbook of self-regulation (pp. 13-40). San Diego, CA: Academic Press.

RIDU / Revista Digital de Investigación en Docencia Universitaria / e-ISSN: 2223-2516

(C) Los autores. Este artículo es publicado por la Revista Digital de Investigación en Docencia Universitaria del Área de Institutional Research and Effectiveness de la Dirección de Aseguramiento de la Calidad, Universidad Peruana de Ciencias Aplicadas. Este es un artículo de acceso abierto, distribuido bajo los términos de la LicenciaCreativeCommons Atribución-CompartirIgual 4.0 Internacional.(http://creativecommons.org/licenses/by-sa/4.0/), que permite el uso no comercial, distribución y reproducción en cualquier medio, siempre que la obra original sea debidamente citada. 